\title{
Inequalities for buckling of a clamped plate
}

\author{
Ü. Ufuktepe \& K. P. Mchale
}

To cite this article: Ü. Ufuktepe \& K. P. Mchale (2002) Inequalities for buckling of a clamped plate, Journal of Interdisciplinary Mathematics, 5:1, 41-47, DOI: 10.1080/09720502.2002.10700305

To link to this article: http://dx.doi.org/10.1080/09720502.2002.10700305

曲 Published online: 31 May 2013.

Submit your article to this journal $\pi$

Џلl Article views: 6

Q View related articles $\sqsubset$ 


\title{
Inequalities for buckling of a clamped plate
}

\author{
Ü. Ufuktepe \\ K. P. Mchale \\ Department of Mathematics \\ Faculty of Sciernce \\ Izmir Institute of Technology \\ Gülbahce-Urla \\ Izmir \\ Turkey
}

ABSTRACT

We study the eigenvalue problems for the buckling of a clamped plate. The previous upper bound on low eigenvalues due to Payne, Pólya, and Weinberger, and Hile and Yeh are reviewed. Using methods similar to those used in bounding ratios of eigenvalues of the membrance problem, bounds for ratios of eigenvalues are found for the buckling of a clamped piate.

\section{INTRODUCTION}

In the present paper, we consider the buckling eigenvalue problem

$$
\left.\begin{array}{ll}
\Delta^{2} u+v \Delta u=0 & \text { in } D \\
u=\frac{\partial u}{\partial n}=0 & \text { on } \partial D
\end{array}\right\}
$$

where $D$ is bounded domain in $\mathbb{R}^{n}$ and $\Delta$ denotes the Laplace operator, $\partial D$ denotes the boundary of $D, n$ denotes the outward normal to $\partial D$, and $v$ denotes eigenvalues of (1.1) and $u$ denotes the corresponding eigenfunction.

Payne, Pólya, and Weinberger [2] considered the problem (1.1) on a bounded domain in $\mathbb{R}^{2}$ and showed that for domains in the plane,

$$
v_{2} \leq 3 v_{1} .
$$

Journal of Interdisciplinary Mathematics

Vol. 5 (2002), No. 1, pp. 41-47

(c) Academic Forum 
Hile and Yeh [1] improved on this and extended it to higher dimensions as

$$
v_{2} \leq \frac{n^{2}+8 n+20}{(n+2)^{2}} v_{1}
$$

Thus, for $n=2$, this gives the better bound

$$
v_{2} \leq 2.5 v_{1} .
$$

Using methods similar to those used in bounding ratios of eigenvalues of the membrane problem, bounds for ratios of eigenvalues are found for the buckling of a clamped plate.

LEMma 1. The quantities $I_{l}=\int_{D} \Psi_{l} \Delta u_{x_{l}}(1 \leq l \leq n)$ satisfy:

(i) $\sum_{l=1}^{n} I_{l}=\frac{n+2}{2}$.

(ii) $\left(\sum_{l=1}^{n} I_{l}\right)^{2} \leq\left(\sum_{l=1}^{n} \int_{D}\left|\nabla \Psi_{l}\right|^{2}\right) \int_{D}(\Delta u)^{2}$.

(iii) $\int_{D} u^{2} \geq \frac{1}{v_{1}}$.

For the vibrating clamped plate problem, Payne, Pólya, and Weinberger and Hile and Yeh use trial functions $\Psi_{i}=x u_{i}-\sum_{j=1}^{k} a_{i j} u_{j}$. With the appropriate choice of $a_{i j}$, the orthogonality conditions necessary to use the Rayleigh-Kitz quotient are met. They both use rotations later in their proofs to simplify the Rayleigh-Ritz inequality. For Theorem 1.2, we would like to use just $\Psi_{i}=x_{i} u_{1}$ as trial functions for $v_{i+1}$ for $i=1, \ldots, n$. However, given an arbitrary choice of Cartesian coordinates $x_{i}$, we have no guarantee that the appropriate orthogonalities (i.e., $\left\langle\Psi_{i}, u_{j}\right\rangle=0$ for all $j \leq i$, where $\langle\cdot, \cdot\rangle$ denotes the appropriate inner product) will hold. To remedy this situation, we argue that we can always find a suitable rotation of axes to a new system of Cartesian coordinates $x_{i}^{\prime}$ so that the desired orthogonalites are obtained (i.e., $\left\langle\widetilde{\Psi}_{i}, u_{j}\right\rangle=\mathbf{0}$ for all $j \leq i$ and $i=1, \ldots, n$ where $\widetilde{\Psi}_{i}=x_{i}^{\prime} u_{1}$ ). Thus the necessary orthogonality conditions will hold for the trial functions $\widetilde{\Psi}_{i}=x_{i}^{\prime} u_{1}$, where the new Cartesian variables $x_{i}^{\prime}$ are obtained via a rotation from our original variables. In other words, 
there exists a real orthogonal matrix $S$ such that $x_{i}^{\prime}=\sum_{j=1}^{n} S_{i j} x_{j}$, for
$i=1, \ldots, n$, and the following theorem holds.

THEOREM 1.1. There exists a set of Cartesian coordinates $x_{i}^{\prime}$ such that the functions $\widetilde{\Psi}_{i}=x_{i}^{\prime} u_{1}$ are suitable trial functions for $v_{i+1}$ in the corresponding Rayleigh quotient. That is, we have

$$
\begin{aligned}
& \widetilde{\Psi}_{i}=\frac{\partial \widetilde{\Psi}_{i}}{\partial n}=0 \text { on } \partial D \\
& \left\langle\widetilde{\Psi}_{\imath}: u_{j}\right\rangle-0, \text { for all } \quad 1 \leq j \leq i \leq n .
\end{aligned}
$$

PROOF. We start from the arbitrary of Cartesian coordinates $x_{i}$ and let $\Psi_{i}=x_{i} u_{1}$. We can assume that these obey $\left\langle x_{i} u_{1}, u_{1}\right\rangle=0$, for, if not, we can simply translate each $x_{i}$ by $a_{i}=\frac{\left\langle x_{i} u_{1}, u_{1}\right\rangle}{\left\langle u_{1}, u_{1}\right\rangle}$. Assuming this has been done, we find that $\widetilde{\Psi}_{i}=\widetilde{x}_{i} u_{1}$ satisfies (1.5) for all $i$ and satisfies (1.6) for $j=1$ and all $i, 1 \leq i \leq n$. To prove (1.6) for $j=2,3, \ldots, i$, let $C$ be an $n \times n$ matrix such that $C=\left[c_{i j}\right]_{1 \leq i \leq j \leq n}$ where $c_{i j}=\left\langle\Psi_{i}, u_{j+1}\right\rangle=\left\langle x_{i} u_{1}, u_{j+1}\right\rangle$.

Then

$$
C=\left[\begin{array}{cccc}
\left.<\Psi_{i}, u_{2}\right\rangle & <\Psi_{i}, u_{3}> & \ldots & <\Psi_{i}, u_{n+1}> \\
<\Psi_{i}, u_{2}> & <\Psi_{i}, u_{3}> & \ldots & <\Psi_{i}, u_{n+1}> \\
\vdots & \vdots & & \vdots \\
\left.<\Psi_{i}, u_{2}\right\rangle & <\Psi_{i}, u_{3}> & \ldots & <\Psi_{i}, u_{n+1}>
\end{array}\right]=\left[\vec{c}_{1}, \vec{c}_{2}, \ldots, \vec{c}_{n}\right]
$$

where the $\vec{c}_{j}$ 's are the columns of $C$. Now using the Gram-Schmidt procedure we can ortogonalize the $\vec{c}_{j}$; $s$ in order, followed by the standard basis vectors $\vec{e}_{i}$ as needed, to get $n$ independent, orthonormal column vectors $\vec{r}_{j}$. Let $R$ be the matrix with columns $\vec{r}_{j}, 1 \leq j \leq n$. Then we have $C=R T$ where $R$ is an $n \times n$ real orthogonal matrix and $T$ is an $n \times n$ upper triangular matrix. Therefore, $R^{T} C=T$ and each entry in the matrix $T$, denoted by $T_{i}$, can be represented as follows:

$$
\begin{aligned}
T_{i j} & \left.=\sum_{k=1}^{n}\left(R^{T}\right)_{i k} c_{k j}=\sum_{k=1}^{n}\left(R^{T}\right)_{i k}<x_{k} u_{1}, u_{j+1}\right\rangle \\
& =\left\langle\left(\sum_{k=1}^{n}\left(R^{T}\right)_{i k} x_{k} u_{1}\right), u_{j+1}\right\rangle=\left\langle x_{k}^{\prime} u_{1}, u_{j+1}\right\rangle .
\end{aligned}
$$


Thus we identify $S$ from our discussion leading up to this theorem as $R^{T}$. Since $T$ is an upper triangular matrix, we have $\left\langle x_{k}^{i} u_{1}, u_{j+1}\right\rangle=0$ for $1 \leq j \leq i, i=2, \ldots, n$. So $\left\langle x_{k}^{\prime} u_{1}, u_{j}\right\rangle=0$ for $2 \leq j \leq i$, and thus $\widetilde{\Psi}_{i}=x_{i}^{\prime} u_{1} \perp u_{2}, u_{3}, \ldots, u_{i}$ for $i=2,3, \ldots, n$. We note also that since $<x_{k} u_{1}, u_{1}>=0$ for $k=1, \ldots, n,<x_{k}^{\prime} u_{1}, u_{1}>=\sum_{k=1}^{n}\left(R^{T}\right)_{i k}<x_{k} u_{1}, u_{1}>=0$ for each $i=1, \ldots, n$. We therefore have $\left\langle\widetilde{\Psi}_{i}, u_{j}\right\rangle=\left\langle x_{k}^{\prime} u_{1}, u_{j}\right\rangle=0$ for $1 \leq j \leq i$ and $i=1, \ldots, n$, which shows that (1.6) is satisfied.

Remark 1. Having established the existence of a suitable system of Cartesian co-ordinates, we revert to our usual notaion $\left(x_{i}\right.$ and $\Psi_{i}$ ) in denoting these objects everywhere aside from this theorem, that is, when using this orthogonality condition outside this theorem, by $x_{i}$ and $\Psi_{i}$ we shall mean $x_{k}^{\prime}$ and $\widetilde{\Psi}_{i}$, respectively, of Theorem 1.1. Also, this orthogonality argument works for the buckling of a clamped plate and vibrating clamped plate problems since the specific inner products of each problems are represented by a general inner product in this theorem.

THEOREM 1.2. (The Main Theorem) Let $v_{1}, v_{2}, \ldots$ denote the successive eigenvalues of the eigenvalue problem below on a bounded domain $D \subset \mathbb{R}^{\prime l}(n \geq 2)$.

$$
\begin{array}{ll}
\Delta^{2} u+v \Delta u=0 & \text { in } D \\
u=\frac{\partial u}{\partial n}=0 & \text { on } \partial D .
\end{array}
$$

Let $u=u_{1}$ denote the eigenfunctioil corresponding to $v_{1}$ normalized so that $\int_{D}|\nabla u|^{2}=1$. Then

$$
\frac{v_{2}+v_{3}+\ldots+v_{n+1}}{v_{1}}<n+4
$$

PROOF. The Rayleigh-Ritz inequality states that

$$
v_{k+1} \leq \frac{\int_{D} \Psi_{k} \Delta^{2} \Psi_{k}}{\int_{D}\left|\nabla \Psi_{k}\right|^{2}}
$$

where $\Psi_{k}$ is any sufficient smooth nontrivial function $\Psi_{k}$, such that 
BUCKLING OF A CLAMPED PLATE

$$
\Psi_{k}=\frac{\partial \Psi_{k}}{\partial n}=0 \quad \text { on } \partial D
$$

and

$$
\int_{D} \nabla \Psi_{k}-\nabla u_{j}=0, \quad j \leq k, \quad k=1, \ldots, n .
$$

Let $\Psi_{k}=x_{k} u$. Then conditions (1.11) are met by virtue of (1.8), and conditions (1.12) are met by an appropriate translation and rotation of the coordinate axes, as detailed in the Theorem 1.1.

Calculating the numerator of (1.10), since $\int_{D} \Psi_{k} \Delta^{2} \Psi_{k}=-\int_{D} U_{1} \Psi_{k} \Delta \Psi_{k}$ $=-v_{1} \int_{D} \Psi_{k} \nabla^{2} \Psi_{k}=v_{1} D\left[\Psi_{k}\right]$, we have

$$
\begin{aligned}
\int_{D} \Psi_{k} \Delta^{2} \Psi_{k} & =\int_{D} \Psi_{k}\left(x_{k} \Delta^{2} u+4 \Delta u_{x_{k}}\right) \\
& =-v_{1} \int_{D} \Psi_{k} x_{k} \Delta u+4 \int_{D} \Psi_{k} \Delta u_{x_{k}} .
\end{aligned}
$$

Using integration by parts and the fact that $D\left[\Psi_{k}\right]=$ $\int_{D} x_{k}^{2}\left|\nabla \Psi_{k}\right|^{2}(D[]$ is Dirichlet integral), we have

$$
\int_{D} \Psi_{k} x_{k} \Delta u=\int_{D}\left|\nabla \Psi_{k}\right|^{2}+\int_{D} u^{2}
$$

Subtitution of (1.13) and (1.14) into (1.10) yields

$$
v_{k+1} \leq \frac{v_{1} \int_{D}\left|\nabla^{2} \Psi_{k}\right|-v_{1} \int_{D} u^{2}+4 \int_{D} \Psi_{k} \Delta u_{x_{k}}}{\int_{D}\left|\nabla \Psi_{k}\right|^{2}}
$$

and hence

$$
v_{k+1}-v_{1} \leq \frac{-v_{1} \int_{D} u^{2}+4 \int_{D} \Psi_{k} \Delta u_{x_{k}}}{D\left[x_{k} u\right]} .
$$

Using (iii) of Lemma 1.1, we have

$$
v_{k+1}-v_{1} \leq \frac{4 \int_{D} x_{k} u \Delta u_{x_{k}}-1}{D\left[x_{k} u\right]} \quad \text { for } k=1, \ldots, n
$$


We need the following:

$$
\begin{aligned}
2 \int_{D} x_{k} u \Delta u_{x_{k}} & =2 \int_{D} \nabla \cdot\left(x_{k} u \Delta u_{x_{k}}\right)-2 \int_{D} \nabla\left(x_{k} u\right) \cdot \nabla u_{x_{k}} \\
& =-2 \int_{D} x_{k} \nabla u \cdot \nabla u_{x_{k}}-2 \int_{D} u u_{x_{k} x_{k}} \\
& =\int_{D}|\nabla u|^{2}+2 \int_{D} u_{x_{k}}
\end{aligned}
$$

and

$$
\left(\int_{D} x_{p} u \nabla u_{x_{k}}\right)^{2}=\left(D\left[x_{k} u, u_{x_{k}}\right]\right)^{2} \leq D\left[x_{k} u\right] D\left[u_{x_{k}}\right]
$$

which implies

$$
\frac{1}{D\left[x_{k} u\right]} \leq \frac{D\left[u_{x_{k}}\right]}{\left(\int_{D} x_{k} u \Delta u_{x_{k}}\right)^{2}}
$$

Substitution of (1.19) into (1.17) yields

$$
\begin{aligned}
v_{k+1}-v_{1} \leq & \frac{2\left(\int_{D}|\nabla u|^{2}+2 \int_{D} u_{x_{k}}^{2}\right)-1}{\left(\int_{D} x_{k} u \Delta u_{x_{k}}\right)^{2}} D\left[u_{x_{k}}\right] \\
= & \frac{4\left(2 \int_{D}|\nabla u|^{2}+4 \int_{D} u_{x_{k}}^{2}-1\right)}{\left(\int_{D}|\nabla u|^{2}+2 \int_{D} u_{x_{k}}^{2}\right)^{2}} D\left[u_{x_{k}}\right] \\
= & \frac{4\left(4 \int_{D} u_{x_{k}}^{2}+1\right)}{\left(1+2 \int_{D} u_{x_{k}}^{2}\right)^{2}} D\left[u_{x_{k}}\right] .
\end{aligned}
$$

Let $a_{k}=\int_{D} u_{x_{k}}^{2}$, then $\sum_{k=1}^{n} a_{k_{k}}=1$ and $0<a_{k}<1$ for $k=1, \ldots, n$. Since we have $\frac{4 a_{k}+1}{\left(1+2 a_{k}\right)^{2}}<1,(1.20)$ implies 


$$
v_{k+1}-v_{1}<4 D\left[u_{x_{k}}\right]
$$

Summing over $k$, we have

$$
v_{2}+v_{3}+\ldots+v_{n+1}-n v_{1}<4 \int_{D}(\Delta u)^{2}
$$

and thus

$$
\frac{v_{2}+v_{3}+\ldots+v_{n+1}}{v_{1}}<n+4
$$

which is (1.9).

\section{REFERENCES}

1. G. N. Hile and R. Z. Yeh, Inequalities for eigenvalues of the biharmonic operator, Pac. J. Math., Vol. 112 (1984), pp. 115-133.

2. L. E. Payne, G. Pólya and H. F. Weinberger, On the ratio of consecutive eigenvalues, J. Math. and Phys., Vol. 35 (1956), pp. 289-298 [reprinted in G. Pólya: Collected Papers, Vol. III: Analysis, J. Hersch and G. C. Rota, editors, MIT

Press, Cambridge, Massachusetts, 1984, pp. 420-429, with comments by $J$. Hersch on pp. 521-522].

Received April, 2000; Revised July, 2000 